\title{
Do heart rate and velocity variability derived from umbilical artery velocity waveforms change prior to clinical pregnancy-induced hypertension?
}

\author{
N. T. C. Ursem, E. B. Clark*, B. B. Keller ${ }^{\dagger}$, W. C. J. Hop $p^{\ddagger}$ and J. W. Wladimiroff
}

Department of Obstetrics and Gynecology, Academic Hospital Rotterdam-Dijkzigt, Erasmus University Rotterdam, The Netherlands; "University of Utah, School of Medicine, Primary Children's Medical Center, Salt Lake City, UT, USA; ${ }^{\dagger}$ Department of Pediatrics, University of Kentucky, Lexington, KY, USA; ${ }^{\ddagger}$ Institute of Epidemiology and Biostatistics, Erasmus University Rotterdam, The Netherlands

Key words: DOPPLER ULTRASOUND, EARLY PREGNANCY, FETAL HEART RATE, CARDIOVASCULAR HOMEOSTASIS, PREGNANCY-INDUCED HYPERTENSION

\begin{abstract}
Objective To investigate the hypothesis that alterations in heart rate variability, peak systolic velocity variability and time-averaged velocity variability in the human umbilical artery may predict early signs of dysfunctional fetalplacental coupling in pregnancies that later develop pregnancy-induced bypertension.
\end{abstract}

Methods Doppler flow velocity recordings from the um-
bilical artery were performed at 10-20 weeks of gestation
in 12 nulliparous women who subsequently developed
pregnancy-induced hypertension. From umbilical artery
velocity waveforms of at least $12 \mathrm{~s}$ in duration we deter-
mined absolute values and beat-to-beat variability in fetal
heart rate, peak systolic and time-averaged velocity and
compared these findings with those in normal nulliparous
pregnant women matched for gestational age.

Results Absolute values for fetal heart rate, peak systolic and time-averaged velocity as well as beat-to-beat variability in fetal heart rate did not differ significantly between women later developing pregnancy-induced bypertension and normal controls. However, variability in peak systolic velocity and time-averaged velocity were decreased in women who subsequently developed pregnancy-induced bypertension.

Conclusions Whereas fetal heart rate variability was similar, umbilical artery flow velocity variability was reduced in women developing pregnancy-induced hypertension compared with controls. It is proposed from this study that variability of the umbilical artery flow velocity is associated with mechanical changes in the vascular bed of women who later develop pregnancy-induced hypertension.

\section{INTRODUCTION}

Hypertensive disorders represent the most common complication during pregnancy, affecting approximately $7 \%$ of all pregnancies ${ }^{1}$. These disorders are the most common cause of fetal growth restriction, and neonatal mortality is high because of prematurity ${ }^{2}$.

Studies using Doppler techniques demonstrate that a high impedance to flow in the uteroplacental circulation in the late second and third trimesters of pregnancy can predict the development of pregnancy-induced hypertension $(\mathrm{PIH})^{3}$ and pre-eclampsia ${ }^{4}$. Abnormal Doppler patterns are associated with defective formation of the placenta, in particular inadequate trophoblast invasion and transforma-

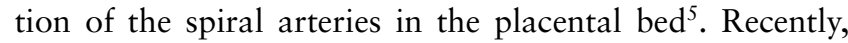
pulsed Doppler investigations of the uteroplacental circulation in the late first and early second trimesters of pregnancy have demonstrated abnormal blood flow indices that are correlated with subsequent development of pregnancy complications $^{6,7}$.

During the past decade spectral analysis of fetal heart rate variability has become a common non-invasive tool to investigate fetal well-being. Metsälä and colleagues ${ }^{8}$ found that an increase in pulsatility index (PI) in the umbilical artery and uterine artery correlated with altered frequencyspecific fetal heart rate variability during the last weeks of

Correspondence: Professor J. W. Wladimiroff, Department of Obstetrics and Gynecology, Academic Hospital Rotterdam-Dijkzigt, Erasmus University, Dr Molewaterplein 40, 3015 GD Rotterdam, The Netherlands 
gestation. A study from our own center on normal umbilical artery flow velocity waveforms at 10-20 weeks of gestation demonstrated gestational age-related changes in short-term variability for both heart rate and absolute flow velocities ${ }^{9}$.

$\mathrm{PIH}$ is often associated with impaired placental perfusion and reduced fetal oxygenation, resulting in centralization of the fetal circulation with redistribution of blood flow to the brain, heart and adrenals ${ }^{10-12}$. The question arises as to whether this process of hemodynamic adaptation is preceded by changes in beat-to-beat regulation of fetal heart rate and umbilical artery flow velocities. Fetal heart rate and flow velocity variability might be measures of cardiovascular function in the human fetus; deviation from the normal could be a specific marker for early cardiovascular dysfunction.

The aim of the present study was to determine whether, at 10-20 weeks of gestation, changes in absolute values and beat-to-beat variability occur for heart rate, peak systolic velocity and time-averaged velocity derived from umbilical artery flow velocity waveforms in women who subsequently develop PIH. Such a marker would be valuable in identifying and guiding therapy for maternal-fetal pairs at risk.

\section{METHODS}

\section{Subjects}

A total of 119 women with a singleton pregnancy were enrolled for umbilical artery flow velocity analysis in a prospective, cross-sectional study at 10-20 weeks of gestation (median 15 weeks). All women were informed and consented to participate in the study, which was approved by the institutional review boards at the Erasmus University Rotterdam and the University of Rochester, NY. All women were nulliparous and did not display any pregnancy pathology at the Doppler survey. In order to obtain a homogeneous group of patients, only nulliparous women were selected. Successful recordings were obtained from 96 of 119 women. Twelve of 96 women developed PIH at 19-35 weeks of gestation (median 28 weeks). PIH was defined as a blood pressure of $140 / 90 \mathrm{mmHg}$ or higher during the second half of pregnancy in a previously normotensive woman. Three women developed pre- eclamptic toxemia at 29, 33 and 34 weeks, respectively. Pre-eclamptic toxemia was defined as PIH in combination with proteinuria of at least $300 \mathrm{mg} / \mathrm{l}$. In two cases, fetal birth weight was below the 10th centile corrected for maternal parity and fetal $\operatorname{sex}^{13}$. Baseline characteristics are given in Table 1.

Twenty-four uneventful nulliparous pregnancies were studied within the same time frame. From our normal population we selected the first two women with a comparable gestational age and parity, who thus served as matched controls. The average value of the clinical and hemodynamic measures of these two normal controls were used as a reference value for the PIH group. These pregnancies resulted in the delivery of a healthy infant with a birth weight between the 10th and 90th centiles corrected for maternal parity and fetal sex ${ }^{13}$.

Pregnancy duration was determined from the last menstrual period and confirmed by ultrasound measurements of fetal crown-rump length (10-12 weeks) or fetal biparietal diameter (13-20 weeks).

\section{Doppler recordings}

Pulsed wave Doppler ultrasound recordings were obtained using a Toshiba SHH-140A machine (Toshiba Corporation, Medical Systems Division, Tokyo, Japan). A combined transvaginal real-time and color Doppler system (carrier frequency $6 \mathrm{MHz}$ and $5 \mathrm{MHz}$, respectively) was used at 10-13 weeks of gestation and a combined transabdominal real-time and color Doppler system (carrier frequency $5 \mathrm{MHz}$ and $3.75 \mathrm{MHz}$, respectively) was used at 14-20 weeks of gestation. The system operated at a power output of less than $100 \mathrm{~mW} / \mathrm{cm}^{2}$ spatial peak-temporal average in both imaging and Doppler modes according to the manufacturer's specifications. Using color Doppler, maximal flow velocity waveforms from the umbilical artery were obtained from a floating loop of the umbilical cord. The angle of insonation was always less than $15^{\circ}$. Sample volume length for all flow velocity waveforms ranged between 0.2 and $0.3 \mathrm{~cm}$; the high-pass wall filter was set at 70-100 Hz. Doppler recordings were performed by one examiner (NTCU). All Doppler studies were carried out with the women in a semirecumbent position and during fetal apnea. Only technically high-quality recordings lasting at least $12 \mathrm{~s}$ were analyzed, to ensure a collection of

Table 1 Baseline characteristics of women developing pregnancy-induced hypertension (PIH) and normal controls. Values are presented as medians, with interquartile ranges in parentheses

\begin{tabular}{lcr}
\hline & $P I H(n=12)$ & Controls $(n=24)$ \\
\hline Gestational age at survey (weeks) & $15(12-18)$ & $15(12-18)$ \\
Maternal age (years) & $29(27-33)$ & $110(100-120)$ \\
Systolic blood pressure at survey $(\mathrm{mmHg})$ & $125(120-150)^{*}$ & $70(70-80)$ \\
Diastolic blood pressure at survey $(\mathrm{mmHg})$ & $80(70-85)$ & $66(62.0-71.5)$ \\
Maternal weight at survey $(\mathrm{kg})$ & $71(66.5-78.5)$ & 2 \\
Smoking during pregnancy $(n)$ & 1 & $38(38-40)$ \\
Gestational age at delivery (weeks) & $39(37-41)$ & $3393(2938-3550)$ \\
Birth weight $(\mathrm{g})$ & $3227(2246-3420)$ & $650(545-675)$ \\
Placental weight $(\mathrm{g})$ & $560(525-638)^{*}$ &
\end{tabular}

*, $p<0.05$ compared to controls 
Table 2 Values presented as medians with interquartile ranges in parentheses for fetal heart rate, peak systolic velocity, time-averaged velocity, heart rate variability, peak systolic velocity variability, time-averaged velocity variability and pulsatility index in the umbilical artery from women developing pregnancy-induced hypertension (PIH) and matched normal controls. Variability data are expressed as standard deviation over a period of at least 25 consecutive heartbeats

\begin{tabular}{lcc}
\hline & $P I H(n=12)$ & Controls $(n=24)$ \\
\hline Heart rate (beats/min) & $152(148-161)$ & $151(147-161)$ \\
Peak systolic velocity $(\mathrm{mm} / \mathrm{s})$ & $320(277-376)$ & $323(246-337)$ \\
Time-averaged velocity $(\mathrm{mm} / \mathrm{s})$ & $186(124-217)$ & $173(129-195)$ \\
Heart rate variability $($ beats/min) & $1.58(1.19-1.80)$ & $1.69(1.29-2.12)$ \\
Peak systolic velocity variability $(\mathrm{mm} / \mathrm{s})$ & $8.54(5.33-11.28)^{*}$ & $12.35(9.40-15.26)$ \\
Time-averaged velocity variability $(\mathrm{mm} / \mathrm{s})$ & $4.69(3.42-7.52)^{*}$ & $6.37(4.76-9.11)$ \\
Pulsatility index & $1.68(1.50-2.84)$ & $1.89(1.47-2.66)$ \\
\hline
\end{tabular}

$*, p<0.05$ compared to controls

at least 25 consecutive waveforms for heart rate and velocity variability analysis. The velocity waveforms (video and hi-fi audio signal) were stored on sVHS videotape in PAL format using a Panasonic model AG7350 machine (Matsushita Electric Ind Co., Japan).

\section{Data processing}

Continuous high-quality Doppler audio waveforms were digitized at $12 \mathrm{kHz}$ using an analogue-to-digital data acquisition board (LabPC+ and BNC-2081 boards, National Instruments, Austin, TX). The maximum flow velocity waveforms from the umbilical artery were reconstructed from the Doppler audio data using computer algorithms developed in our center using LabVIEW software (National Instruments, Austin, TX). A detailed description of the analysis technique and the computer algorithms has been published previously ${ }^{14}$. Umbilical artery peak systolic velocity $(\mathrm{mm} / \mathrm{s})$, time-averaged velocity $(\mathrm{mm} / \mathrm{s})$ and instantaneous heart rate (beats/min) for each cardiac cycle were calculated. For the entire maximum velocity waveform the mean and standard deviation for peak systolic velocity, time-averaged velocity and heart rate were calculated to establish beat-to-beat variability for each of these variables. The umbilical artery PI was calculated from the difference between the maximal and minimal flow velocity divided by the time-averaged velocity.

A previous reproducibility study of the umbilical artery flow velocity waveform recordings at 10-20 weeks of gestation revealed a mean coefficient of variation $(\mathrm{CV})$ of $1.1 \%$ (range $0.2-3.5 \%$ ) for fetal heart rate, a mean CV of $1.9 \%$ (range $1.1-3.3 \%$ ) for peak systolic velocity, and a mean $\mathrm{CV}$ of $2.4 \%$ (range $1.2-3.9 \%$ ) for time-averaged velocity ${ }^{9}$.

\section{Statistical analysis}

Data are presented as median and interquartile range. Since the normal umbilical artery flow velocity waveform at 10-20 weeks of gestation demonstrated gestational agerelated changes in variability for both heart rate and absolute flow velocities ${ }^{9}$, a paired Wilcoxon's test was used. The controls were matched for gestational age and parity. Baseline characteristics and hemodynamic parameters were compared between women developing PIH and matched normal controls. Statistical significance was reached at $p<0.05$. Calculations were performed with SPSS 6.1 software (SPSS Inc., Chicago, IL).

\section{RESULTS}

Women developing PIH $(n=12)$ displayed a higher systolic blood pressure at the Doppler survey and a lower placental weight compared to normal controls (Table 1). The median time interval between Doppler survey and onset of clinical PIH was 13 weeks (range 1-25 weeks). The fetal heart rate, umbilical artery peak systolic velocity, time-averaged velocity and PI did not differ significantly between the two study groups (Table 2). Beat-to-beat variability in fetal heart rate did not differ significantly between women developing PIH and normal controls, but beat-to-beat variability in umbilical artery peak systolic and time-averaged velocity was lower in the study group (Table 2 ). The individual data for the hemodynamic parameters measured in the umbilical artery in fetuses of women who later developed PIH and normal controls are depicted in Figure 1.

\section{DISCUSSION}

The present study aimed at determining whether changes in beat-to-beat variability of fetal heart rate and umbilical artery flow velocities could detect fetal placental dysfunction early in pregnancies that later developed PIH. PI calculations have demonstrated that uncomplicated pregnancies are characterized by a gradual gestational age-related decrease in umbilical-placental downstream impedance starting from $12-13$ weeks of gestation ${ }^{15,16}$. It was recently noted that heart rate variability and velocity variability in normal umbilical artery flow velocity waveforms are related to gestational age ${ }^{9}$. An increase in heart rate variability at 15-20 weeks suggests a correlation with functional maturation of the parasympathetic nervous system, whilst an increase in flow velocity variability may reflect a cardiovascular feedback control mechanism similar to that in the chick embryo. In the preinnervated chick embryo, aortic blood flow velocity and vascular impedance are amplitude-modulated ${ }^{17}$. These modulations are associated with hemodynamic regulation prior to the operation of autonomic control mechanisms. The mechanism for hemodynamic regulation prior to autonomic innervation is still unknown ${ }^{18}$. 

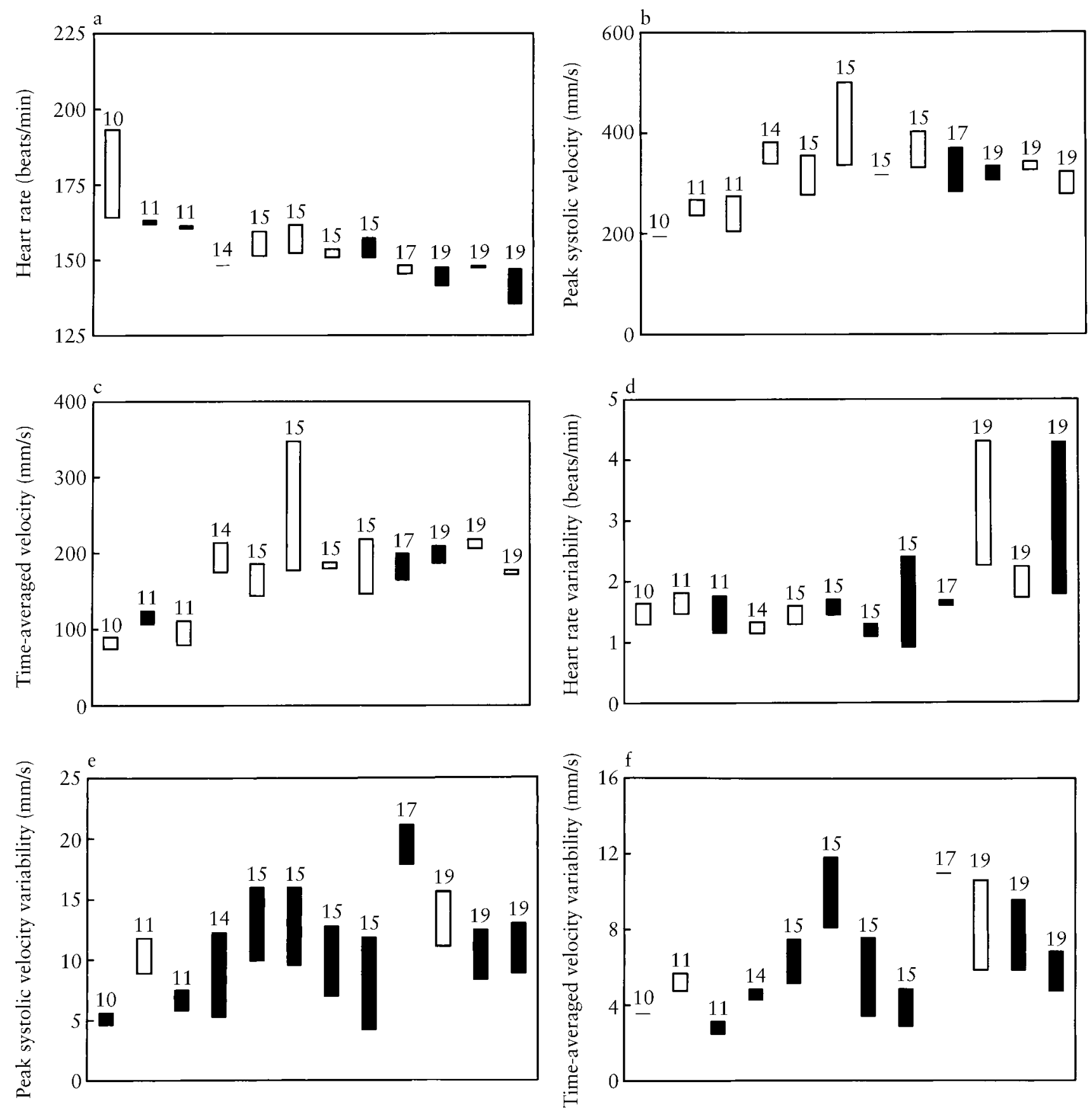

Figure 1 Individual data of hemodynamic parameters measured in the umbilical artery from women developing pregnancy-induced hypertension $(\mathrm{PIH})(n=12)$ and normal pregnant controls $(n=24)$ as a function of gestational age (weeks). Solid boxes indicate that a particular value measured in women developing PIH was lower compared with normal controls and open boxes indicate that a particular value measured in women developing PIH was higher compared with normal controls. The data labels above the boxes indicate the gestational age. The difference between women developing PIH and normal controls are depicted for heart rate (a), peak systolic velocity (b), time-averaged velocity (c), heart rate variability (d), peak systolic velocity variability (e) and time-averaged velocity variability (f). Note that both peak systolic velocity variability (d) and time-averaged velocity variability (e) were significantly decreased in women developing PIH compared with normal controls

Histological studies of the placental bed of pregnancies complicated by hypertension show inadequate trophoblast invasion and transformation of the spiral arteries of the placental bed in early pregnancy ${ }^{5,19}$. In cases of PIH the physiological vascular changes are limited to some spiral arteries in the decidual segments with some remaining in the same state as spiral arteries in the non-pregnant uterus $^{20}$. Biagini and co-workers ${ }^{21}$ showed that in placentae of hypertensive women there is a higher number of chorionic villi-decidua interactions and a smaller surface area of a single interaction than in the placentae of normotensive women. Thus, the placental vascular interface between mother and fetus may be abnormal prior to the development of clinical PIH. 
Based on these histological data the search began for early hemodynamic data from the uteroplacental and fetal circulations as predictors of $\mathrm{PIH}$ and/or fetal growth restriction developing later in pregnancy. Uterine artery flow velocity waveforms depicting reduced end-diastolic flow velocities and early diastolic notching as early as 18-20 weeks of gestation were predictive of subsequent development of $\mathrm{PIH}^{4}$. Even at 12-13 weeks of gestation Doppler-detectable changes are established in the uterine artery that are associated with subsequent development of pregnancy complications ${ }^{6,7}$. Contradictory findings were observed in the umbilical artery flow velocity waveform at this early stage of pregnancy. In one study no changes were observed in umbilical blood flow velocity waveforms prior to the emergence of $\mathrm{PIH}^{22}$. In another study, by contrast, abnormal Doppler values in the umbilical artery were associated with premature delivery, the development of PIH and the delivery of a small-forgestational-age baby during the late second or third trimester of pregnancy ${ }^{7}$.

In the present study there was a decrease in umbilical artery flow velocity variability, but the absolute peak systolic and time-averaged velocity were similar for women developing PIH and normal controls. Moreover, the placental weight of women developing PIH was decreased compared to normal controls. Increased arterial downstream impedance due to abnormal morphological changes of the vascular bed of the placenta may be responsible for the decrease in velocity variability. However, the absence of a difference in umbilical artery PI values between fetuses of women developing PIH and normal controls argues against a modification in afterload (Table 2). Alternatively, changes in atrial compliance may occur without altering afterload. An in vitro study has shown that the concentration of lipoperoxide in the microvillous membrane of women who subsequently developed PIH was increased over that of normotensive pregnant women ${ }^{23,24}$. Lipoperoxidation products are capable of inducing smooth muscle constriction $^{24}$ and increase the responsiveness to angiotensin $\mathrm{II}^{25}$. It is proposed that this will result in a reduced compliance of the placental vessels and therefore reduction in umbilical artery flow velocity variability in women, who later develop PIH.

In the present study beat-to-beat variability in fetal heart rate was similar between women developing $\mathrm{PIH}$ and normal controls. This suggests that parasympathetic activity, which plays an important role in the control of heart rate variability, is not altered in women who develop $\mathrm{PIH}$ at a later stage.

The patients enrolled in the present study represent those with a subset of PIH pregnancies with nearly normal fetal development and outcome. Even in this subset of pregnancies we were able to detect differences between the two populations. The small number of patients in this study means that the results must be interpreted with care. The size of our study allows the discrimination between means of approximately one standard deviation. Power spectral analysis of longer lasting umbilical artery waveform recordings will be needed to clarify which mecha- nisms are involved in the observed cardiovascular changes in women who later develop PIH.

It can be concluded that beat-to-beat variability in the fetal heart rate was similar for women developing PIH and normal controls. However, a significant decrease in umbilical artery flow velocity variability was observed in women developing PIH. Changes in the uteroplacental circulation ${ }^{7}$ and biochemical changes ${ }^{25}$ may be associated with the observed decrease in variability in umbilical artery blood flow velocity.

\section{ACKNOWLEDGEMENT}

This study is supported by P50-HL51498, the SCOR in Pediatric Cardiovascular Disease, at the University of Rochester, Rochester, NY.

\section{REFERENCES}

1. Chesley LC, ed. Hypertension Disorders in Pregnancy. New York: Appleton Century-Crofts, 1978

2. Sibai BM, Abdella TN, Anderson GD. Pregnancy outcome in 211 patients with mild chronic hypertension. Obstet Gynecol 1983;61:571-6

3. Steel SA, Pearce JM, McParland P, Chamberlain GVP. Early Doppler ultrasound screening in prediction of hypertensive disorders of pregnancy. Lancet 1990;335:1548-51

4. Bower S, Schuchter K, Campbell S. Doppler ultrasound screening as part of routine antenatal scanning: prediction of pre-eclampsia and intrauterine growth retardation. $\mathrm{BrJ} \mathrm{Ob}$ stet Gynaecol 1993;100:989-94

5. Robertson WB, Khong TY, Brosens I, De Wolf F, Sheppard BL, Bonnar J. The placental bed biopsy: review from three European centers. Am J Obstet Gynecol 1986;155:401-12

6. van den Elzen HJ, Cohen-Overbeek TE, Grobbee DE, Quartero RW, Wladimiroff JW. Early uterine artery Doppler velocimetry and the outcome of pregnancy in women aged 35 years and older. Ultrasound Obstet Gynecol 1995;5:328-33

7. Harrington K, Goldfrad C, Carpenter RG, Campbell S. Transvaginal uterine and umbilical artery Doppler examination of 12-16 weeks and the subsequent development of pre-eclampsia and intrauterine growth retardation. Ultrasound Obstet Gynecol 1997;9:94-100

8. Metsälä TH, Pirhonen JP, Jalonen JO, Erkkola RU, Välimäki IAT. Association of abnormal flow velocity waveforms in the uterine artery with frequency-specific fetal heart rate variabilty. Early Hum Dev 1993;34:217-25

9. Ursem NTC, Struijk PC, Hop WCJ, Clark EB, Keller BB, Wladimiroff JW. Heart rate and flow velocity variability as determined from umbilical Doppler velocimetry at 10-20 weeks of gestation. Clin Sci 1998;95:539-45

10. Wladimiroff JW, Tonge HM, Stewart PA. Doppler ultrasound assessment of cerebral blood flow in the human fetus. $\mathrm{Br} \mathrm{J}$ Obstet Gynaecol 1986;93:471-5

11. Chaoui R. The fetal 'heart-sparing effect' detected by the assessment of coronary blood flow: a further ominous sign of fetal compromise? Ultrasound Obstet Gynecol 1996;7:5-9

12. Mari G, Uerpairojkit B, Abuhamad AZ, Copel JA. Adrenal artery velocity waveforms in the appropriate and small-forgestational-age fetus. Ultrasound Obstet Gynecol $1996 ; 8: 82-6$

13. Kloosterman G. On intrauterine growth. Int J Obstet Gynaecol 1970;8:895-912

14. Ursem NTC, Brinkman HJF, Struijk PC, Hop WCJ, Kempski $\mathrm{MH}$, Keller BB, Wladimiroff JW. Umbilical artery waveform analysis based on maximum, mean and mode velocity in early human pregnancy. Ultrasound Med Biol 1998;24:1-7 
15. van Splunder IP, Stijnen T, Wladimiroff JW. Fetal atrioventricular flow-velocity waveforms and their relation to arterial and venous flow-velocity waveforms at 8 to 20 weeks of gestation. Circulation 1996;94:1372-8

16. Wladimiroff JW, Huisman TW, Stewart PA. Fetal cardiac flow velocities in the late 1st trimester of pregnancy: a transvaginal Doppler study. J Am Coll Cardiol 1991;17:1357-9

17. Kempski MH, Kibler N, Blackburn JL, Dzakowic J, Hu N, Clark EB. Hemodynamic regulation in the chick embryo. ASME Adv Bioeng 1993;24:119-22

18. Kempski MH. Modeling and control of embryonic hemodynamics. In Clark EB, Markwald RR, Takao A, eds. Developmental Mechanisms of Heart Disease. Armonk: Futura Publishing, 1995:421-34

19. Pijnenborg R, Anthony J, Davey DA, Rees A, Tiltman A, Vercruysse L, van Assche A. Placental bed spiral arteries in the hypertensive disorders of pregnancy. Br J Obstet Gynaecol 1991;98:648-55

20. Khong TY, De Wolf F, Robertson WB, Brosens I. Inadequate maternal vascular response to placentation in pregnancies complicated by pre-eclampsia and by small-for-gestational age infants. Br J Obstet Gynaecol 1986;93:1049-59
21. Biagini G, Pugnaloni A, Carboni V, Mazzanti L, Cester N, Romanini C, Toschi E, Castaldini C. Placental villi-decidua interactions in normal and hypertensive pregnancies: a morphological quantitative study. Gynecol Obstet Invest 1992;34:15-19

22. Arduini D, Rizzo G, Romanini C. Doppler ultrasonography in early pregnancy does not predict adverse pregnancy outcome. Ultrasound Obstet Gynecol 1991;1:180-5

23. Cester N, Staffolani R, Rabini RA, Magnanelli R, Salvolini E, Galassi, R, Mazzanti L, Romanini C. Pregnancy induced hypertension: a role for peroxidation in microvillus plasma membranes. Mol Cell Biochem 1994;131:151-5

24. Gurtner GH, Knoblauch A, Smith PL, Sies H, Adkinson NF. Oxidant- and lipid-induced pulmonary vasoconstriction mediated by arachidonic acid metabolites. J Appl Physiol 1983;55: 949-54

25. Hubel CA, Roberts JM, Taylor RN, Musci TJ, Rogers GM, McLaughlin MK. Lipid peroxidation in pregnancy: new perspectives on preeclampsia. Am J Obstet Gynecol 1989;161: $1025-34$ 